\section{Factores de riesgo para el desarrollo de infecciones intrahospitalarias en pacientes quemados. Hospital S. Juan de Dios, Costa Rica: Enero 2003-Diciembre 2005. Parte I Risk factors for development of intrahospitalary infections in hurned patients. Hospital S. Juan de Dios, Costa Rica: January 2003-December 2005. PartI}

\author{
Vargas Naranjo, S.*, Romero Zúñiga, J J .**, Prada Castellanos, Y.***, \\ Fonseca Portilla, G.***, Lao Gallardo, W.***k
}

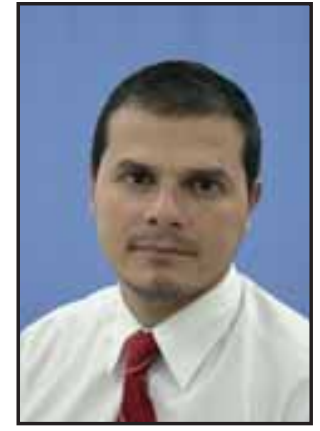

Vargas Naranjo, S.

\section{Resumen}

La infección intrahospitalaria es una de las principales causas de morbilidad y mortalidad en los pacientes quemados. El presente estudio caracteriza a los ingresados por quemadura(s) y la presencia de esta clase de infección en la Unidad Nacional de Quemados del Hospital San Juan de Dios, en Costa Rica, entre los años 2003 y 2005.

Realizamos un análisis descriptivo de la infección intrahospitalaria en el sitio de la quemadura según las características de los pacientes y de la quemadura así como el total de días de estancia hospitalaria, utilizando para ello un diseño de estudio tipo cohorte retrospectiva.

La incidencia acumulada de la infección intrahospitalaria en el sitio de la quemadura fue de $19.0 \%$. Los meses posteriores al ingreso a clases presentaron un mayor número de hospitalizaciones por quemaduras. La edad, la superficie corporal total quemada, el número de segmentos corporales quemados, la profundidad de la quemadura y los días de estancia hospitalaria presentaron diferencias significativas entre los individuos infectados y no infectados. El tipo de accidente que produjo la quemadura y el sexo no presentaron diferencias significativas entre ambos grupos de pacientes. La electricidad fue el agente causal más frecuente en los pacientes con infección, mientras que el agua caliente lo fue en los pacientes sin infección.

Concluimos que las características fisiopatológicas de las quemaduras favorecen infección en los pacientes quemados; asimismo, existen factores que pueden y deben ser tomados en cuenta para controlar y prevenir la infección y sus efectos.

\begin{tabular}{|c|c|}
\hline Palabras clave & $\begin{array}{l}\text { Infección nosocomial, Profundidad de la } \\
\text { quemadura, Superficie corporal total } \\
\text { quemada. }\end{array}$ \\
\hline Código numéric & $154-1541-1546$ \\
\hline
\end{tabular}

Intrahospitalary infection in burned patients is one of the most important causes of morbidity and mortality. This study characterizes the admitted burn victims and the presence of inner-hospital infection at the National Burn Victims Unit, San Juan de Dios Hospital, Costa Rica, between 2003 and 2005.

We made a descriptive analyze of intrahospital infection in the burned area according characteristics of the patients and their burn(s), as well as the total number of days of hospital stay, using a prospective cohort.

The accumulated incidence for intra hospital infection in the burned area was $19.0 \%$. In the months following the beginning of the school year, the number of burn victims admitted in the hospital increased. Age, total burnt surface, the number of burnt body segments, the depth of the burn and the days of hospital stay varied significantly between infected and not infected individuals. The cause of the burn and the victim's gender did not reveal any relevant differences between the groups of patients. For patients that developed intrahospital infection in the burned area, electricity was the most frequent causing agent, as was hot water for patients without intra hospital infection in the burned area.

As a conclusion, physiopathologic characteristics of the burns favor the occurrence of the intra hospital infection in the burned area; furthermore, there are factors than can and must be taken into account to control and prevent intrahospital infection in the burned area and its effects.

$\begin{array}{ll}\text { Key words } & \text { Bursns, Nosocomial infection, Burn } \\ \text { depth, Total burn surface }\end{array}$

Numeral Code 154-1541-1546

\footnotetext{
* Epidemiólogo. Asistente de Auditoría Interna. Caja Costarricense de Seguro Social.

* Epidemiólogo. Profesor del Postgrado en Epidemiología. Universidad Nacional, Costa Rica.

* Asistente del Servicio de Cirugía Plástica y Reconstructiva. Hospital San J uan de Dios. Costa Rica.

* Odontólogo-Epidemiólogo de la Dirección Regional de Servicios de Salud. Caja Costarricense de Seguro Social.

Unidad de Epidemiología y Unidad Nacional de Quemados, Hospital San J uan de Dios. Costa Rica.
} 


\section{Introducción}

Como se sabe, las quemaduras se clasifican de acuerdo a la cantidad de superficie corporal afectada y la profundidad del daño, siendo la primera el principal criterio para la evaluación inicial del paciente. Para evaluar la superficie corporal total quemada (SCTQ), de forma práctica y rápida, en pacientes adultos se utiliza con frecuencia la regla de los 9 de Wallace (1).Por otra parte, la profundidad de la quemadura (PQ) depende de la temperatura, densidad y $\mathrm{pH}$ del agente causante y del tiempo de exposición a él $(1,2)$.

Los agentes causales más frecuentemente citados en la literatura mundial son el fuego, la electricidad, los objetos o líquidos calientes y ciertos productos químicos, elementos que pueden causar desde trastornos leves hasta lesiones graves que ponen en peligro la vida $(1,3)$. Los factores de riesgo asociados y sus agentes causales, varían con la edad de los individuos y su entorno sociocultural $(3,4)$; así, en algunos países se ha observado que los niños se queman más que los adultos $(2,5)$.

Las estadísticas sobre quemados son imprecisas; dependiendo de la fuente, el número anual de quemados puede llegar, incluso, a más de dos millones en Estados Unidos (6); se estima que, durante un año de calendario, el $1 \%$ de la población sufre una quemadura que requiere tratamiento por un profesional de salud. En países como Colombia, el $60 \%$ de las quemaduras se presenta en el género masculino y en los niveles sociales bajos $(2,3,7)$. En Costa Rica, la incidencia de pacientes quemados que ingresa a la Unidad Nacional de Quemados del Hospital San Juan de Dios (UNQ-HSJD) es aproximadamente de 150 a 175 personas por año (8). Estudios realizados en Israel han confirmado que las quemaduras en general, por su evolución, requieren más atención por parte del personal de salud que otros tipos de trauma (9). Adicionalmente, se ha visto que la mayoría de los fallecimientos ocurren en la primera semana tras la quemadura, cuando la infección es el predictor más importante de este desenlace, junto con las lesiones causadas por inhalación de productos tóxicos $(10,11)$.

Las infecciones intrahospitalarias (IIH), por definición, se presentan después de las primeras 48-72 horas de estancia en el hospital y no deben estar presentes o en incubación en el momento del ingreso $(12,13)$. Hay que tener en cuenta que el período de 48-72 horas que se utiliza para diferenciar las IIH y las adquiridas en la comunidad es un criterio general. Algunas IIH pueden presentarse antes, particularmente cuando se asocian a procedimientos invasivos; están documentadas bacteriemias nosocomiales que se presentan antes de las 24 primeras horas de internamiento (12). En pacientes quemados, las $\mathrm{IIH}$ son muy frecuentes con una tasa de 36.2/1000 pacientes por día, provocando el $75 \%$ de los fallecimientos en pacientes quemados $(10,13)$. La incidencia acumulada de IIH en pacientes quemados entre los años 2002 y 2005 en un estudio descriptivo realizado en el Hospital San Juan de Dios sobre una muestra de 104 pacientes, fue del $60 \%$; sin embargo, la proporción observada por los profesionales que brindan atención médica a los pacientes quemados en ese centro hospitalario es menor, de hasta de un 40\% (Prada, 2006, comunicación personal).

Debido a que en las quemaduras hay tejidos descubiertos y desvitalizados, los pacientes quemados son más susceptibles a la colonización por bacterias (13). La edad, los procedimientos quirúrgicos asociados, la SCTQ, la PQ y el número de segmentos corporales quemados (NSCQ), son algunos factores que favorecen el desarrollo de IIH en pacientes quemados; también hay que tener en cuenta la existencia de enfermedades concomitantes, como el virus de la inmunodeficiencia humana $(2,14,15)$.

El presente estudio tiene como objetivo caracterizar la infección intrahospitalaria en el sitio de la quemadura (IISQ) en los pacientes ingresados en la UNQHSJD entre los años 2003 y 2005.

\section{Material y método}

Realizamos un estudio de cohorte-retrospectivo, en la UNQ-HSJD, Costa Rica, entre el 1 de enero de 2003 y el 31 de diciembre de 2005. Incluimos todos los pacientes quemados ingresados en dicha Unidad, mayores de 12 años, sin importar el sexo, la raza, la procedencia o la nacionalidad. Excluimos aquellos pacientes con una estancia hospitalaria menor de 48 horas, así como los que presentaron una estancia hospitalaria mayor, pero con algún tipo de infección existente al momento de su ingreso; también aquellos que presentaron enfermedades o condiciones, que por su naturaleza, pudieran influir en la presentación y en la recuperación de las infecciones, (diabetes mellitus, HIV/SIDA) o compromiso del sistema inmunológico.

Analizamos la presencia de IISQ según sexo, agente causal que produjo la quemadura (electricidad, fuego directo, agua caliente, otros), número de segmentos corporales quemados (NSCQ), tipo de accidente (ocupacional, domiciliar, otros), PQ (grados 1, 2 y 3), SCTQ, edad en años (13 a 19, 20 a 39, 40 a 64 y > 64) el tiempo en de estancia hospitalaria en días (DEH) y los días de estancia hospitalaria previos al desarrollo de la IISQ (DEHP-IISQ).

Para categorizar el NSCQ, el cuerpo humano se dividió en segmentos: a) cabeza y cuello, b) tórax y abdomen, c) extremidades superiores, d) extremidades inferiores y e) genitales y glúteos. Adicionalmente, se estableció si el paciente sufrió quemaduras solamente en uno, en 2 ó en más segmentos corporales. 
Tuvimos en cuenta la clasificación de Boyer para la PQ (1); sin embargo, para este estudio se incluyó en el grado 3, el grado 4, que Boyer clasifica como aquellas quemaduras cuyo daño se extiende a estructuras profundas (músculos, tendones y hueso).

Consideramos IISQ a toda infección diagnosticada e incluida en el expediente clínico por un médico, que apareciera tras las primeras 48 horas posteriores al ingreso del paciente a la UNQ-HSJD, o bien en un tiempo menor al anterior, pero con agentes patógenos multirresistentes identificados mediante cultivo positivo y prueba de resistencia antimicrobiana.

Para la captura de los datos empleamos un cuestionario físico y digital. El análisis se llevó a cabo con los programas Epiinfo versión 3.3.2, Egret 2.0.31 y Excel versión 8.0. Evaluamos la integridad de la base de datos creada examinando cada variable por separado y observando que cada valor fuera coherente con los cuestionarios originales; adicionalmente, eliminamos los registros incompletos en los que 2 o más variables no hubieran sido registradas.

Para las variables continuas como edad, SCTQ, DEH y DEHP-IISQ, calculamos el promedio con su intervalo de confianza al $95 \%\left(\mathrm{IC}_{95 \%}\right.$ ), además de su rango. Para las variables NSCQ, PQ, tipo de accidente, agente causal, edad (categorizada) y sexo, calculamos las relativas.

La normalidad de los datos para las variables cuantitativas fue evaluada por medio de la prueba Shapiro
Wills modificada; posteriormente, realizamos pruebas de hipótesis para diferencia de medias ( $\mathrm{t}$ de student), mientras que para las variables cualitativas utilizamos la prueba Chi-cuadrado, ambas con un valor $\alpha=0.05$.

\section{Resultados}

Los registros hospitalarios reportaron un total de 552 ingresos a la UNQ-HSJD entre enero de 2003 y diciembre de 2005. Todos los registros evaluados cumplieron los criterios de inclusión; de ellos, 211 cumplieron los criterios de exclusión, quedando disponibles 341 registros para el estudio. De lellos, 77 $(17,8 \%)$ sufrieron $\mathrm{IIH}$, de los cuales $65(84,4 \%)$ presentaron IISQ. La incidencia acumulada para la IISQ fue de $19.0 \%$.

$\mathrm{Al}$ analizar la casuística mensual de los ingresos por quemaduras con 2 o más días de internamiento, determinamos un aumento de casos en los meses de julio y agosto de 2003, con una tendencia bimodal en el año 2004, en los meses de febrero-marzo y julio-agosto. Asimismo, en el 2005 observamos un aumento de casos en los meses de febrero y marzo con una disminución en el segundo semestre del año (Fig. 1). Observamos que no hay una clara tendencia espacial en la presentación de las IISQ. Las tasas por 100 pacientes quemados ingresados en la UNQ-HSJD por bimestre, mostraron un aumento en el número de IISQ en el año 2005 en comparación con los años anteriores (Fig. 2).
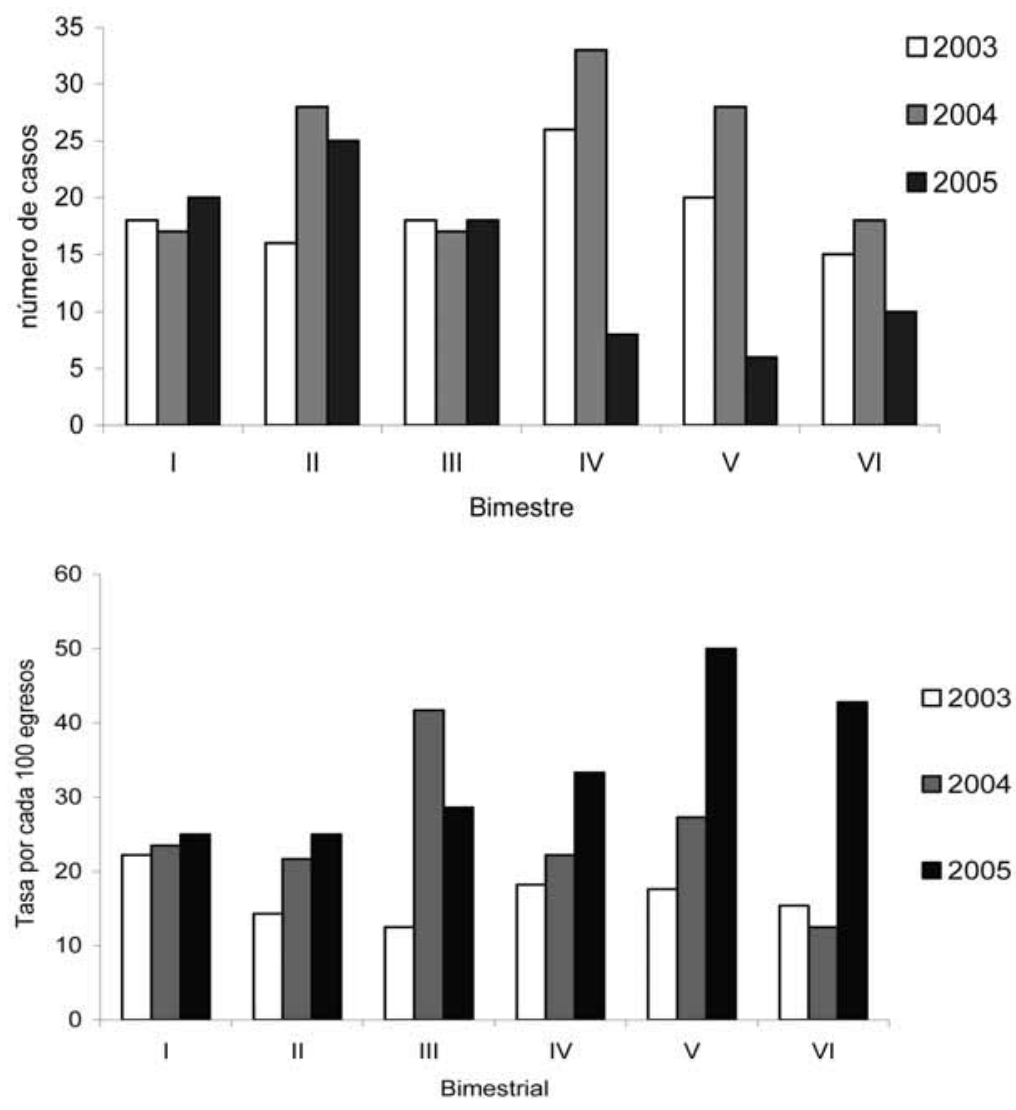

Fig. 1. Distribución temporal (bimestral) de los ingresados por quemadura(s) en la UNQ-HSJ D. Enero de 2003 a diciembre de 2005.
Fig. 2. Distribución temporal (bimestral) de las tasas de IISQ por cada 100 ingresados por quemadura(s) en la UNQ-HSJ D. Enero de 2003 a diciembre de 2005. 
Tabla I. Descripción de las variables: edad, superficie corporal total quemada, días de estancia hospitalaria total y días de estancia previa a la IISQ, de los ingresados por quemadura(s) en la UNQ-HSJD. Enero 2003 a diciembre de 2005

\begin{tabular}{|c|c|c|c|c|c|c|c|}
\hline \multirow[b]{2}{*}{ Variable } & \multicolumn{3}{|c|}{$\sin I I S Q$} & \multicolumn{3}{|c|}{ con IISQ } & \multirow[t]{2}{*}{ t-student } \\
\hline & $\mathrm{n}^{*}$ & $\stackrel{\mu}{\mu}$ & rango & $n^{*}$ & $\stackrel{\mu}{\mu}$ & rango & \\
\hline Edad & 276 & $\begin{array}{c}33.3 \\
(27.7-38.9)\end{array}$ & 70 & 64 & $\begin{array}{c}36.3 \\
(29.7-42.9)\end{array}$ & 79 & $p=0.184$ \\
\hline SCTQ & 241 & $\begin{array}{c}17.0 \\
(11.4-22.6)\end{array}$ & 98 & 62 & $\begin{array}{c}34.4 \\
(27.8-41.0)\end{array}$ & 77 & $p<0.001$ \\
\hline DEH & 276 & $\begin{array}{c}8.8 \\
(5.5-12.1)\end{array}$ & 55 & 65 & $\begin{array}{c}33.6 \\
(26.8-40.4)\end{array}$ & 71 & $p<0.001$ \\
\hline DEHP-IISQ & & & & 62 & $\begin{array}{c}10.1 \\
(6.4-13.8)\end{array}$ & 44 & \\
\hline
\end{tabular}

Observamos que los individuos de 20 a 64 años son los que más frecuentemente se queman (Fig. 3). No encontramos diferencias significativas en el promedio de edades de los grupos de infectados y no infectados; no obstante, sí observamos diferencias entre la frecuencia de los estratos por edad y determinamos que el grupo de individuos mayormente afectados por IISQ fue el de los mayores de 64 años (Tablas I y II).
El promedio de SCTQ en los pacientes sin IISQ fue de $17.0 \%$ ( $\mathrm{IC}_{95 \%} 11.4-22.6 \%$ ), mientras que para los pacientes con IISQ fue mayor (Tabla I).

Para los pacientes sin IISQ, el promedio DEH fue de 8.8 días ( $\mathrm{IC}_{95 \%}$ 5.5-12.1 días), mientras que para los pacientes con IISQ, fue mayor; a este último grupo se le calculó el promedio de DEHP-IISQ, que tuvo un valor de 10.1 días (Tala I).

Tabla II. Proporción de los pacientes ingresados por quemadura(s) en la UNQ-HSJD, según grupo de edad. Enero 2003 a diciembre de 2005

\begin{tabular}{|c|c|c|c|c|c|c|}
\hline \multirow[b]{2}{*}{ Edad (años) } & \multicolumn{3}{|c|}{ Sin IISQ } & \multicolumn{3}{|c|}{ con IISQ } \\
\hline & $\mathrm{n}$ & $\begin{array}{c}\mathrm{a} \\
\left(\mathrm{IC}_{95 \%}\right)\end{array}$ & $\begin{array}{c}\mathrm{b} \\
\left(\mathrm{IC}_{95 \%}\right)\end{array}$ & $\mathrm{n}^{*}$ & $\begin{array}{c}\mathrm{a} \\
\left(\mathrm{IC}_{95 \%}\right)\end{array}$ & $\begin{array}{c}\mathrm{B} \\
\left(I \mathrm{C}_{95 \%}\right)\end{array}$ \\
\hline 13 a 19 & 48 & $\begin{array}{c}85.7 \\
(76.0-89.5)\end{array}$ & $\begin{array}{c}17.4^{*} \\
(13.2-22.2)\end{array}$ & 8 & $\begin{array}{c}14.3 \\
(6.5-25.8)\end{array}$ & $\begin{array}{c}12.5^{*} \\
(5.6-22.9)\end{array}$ \\
\hline 20 a 39 & 144 & $\begin{array}{c}81.8 \\
(75.6-84.6)\end{array}$ & $\begin{array}{c}52.2^{*} \\
(46.3-56.9)\end{array}$ & 32 & $\begin{array}{c}18.2 \\
(12.9-24.4)\end{array}$ & $\begin{array}{c}50.0^{*} \\
(38.0-60.1)\end{array}$ \\
\hline 40 a 64 & 74 & $\begin{array}{c}78.7 \\
(70.1-83.0)\end{array}$ & $\begin{array}{c}26.8^{*} \\
(21.8-32.1)\end{array}$ & 20 & $\begin{array}{c}21.3 \\
(13.7-30.4)\end{array}$ & $\begin{array}{c}31.2^{*} \\
(20.6-42.8)\end{array}$ \\
\hline$>64$ & 10 & $\begin{array}{c}71.4 \\
(47.4-84.4)\end{array}$ & $\begin{array}{c}3.6^{\prime} \\
(1.8-6.6)\end{array}$ & 4 & $\begin{array}{c}28.6 \\
(9.2-54.8)\end{array}$ & $\begin{array}{c}6.3^{\nu} \\
(1.8-15.2)\end{array}$ \\
\hline
\end{tabular}

* Solo 64 casos de las IISQ reportaron la edad de los pacientes.

$\mu=$ Promedio, $I C_{95 \%}=$ Intervalo de Confianza al 95\%, a = porcentaje de fila, $b=$ porcentaje de columna. Los signos en superindice representan los resultados de las pruebas de hipótesis intraestrato para la frecuencia en la fila, entre los individuos de cada grupo, con $\alpha=0.05$ como estadístico de prueba.

Fuente: Expedientes Clínicos, Hospital San Juan de Dios. 
Tabla III. Descripción de las variables sexo, agente causal, tipo de accidente, NSCQ y PQ en los ingresados en la UNQ-HSJD. Enero 2003 a diciembre de 2005

\begin{tabular}{|c|c|c|c|}
\hline Variable & Categoría & $\begin{array}{c}\text { Sin IISQ } \\
\text { n (\%) } \\
{\left[\mathrm{IC}_{95 \%}\right]}\end{array}$ & $\begin{array}{c}\text { Con IISQ } \\
\text { n (\%) } \\
{\left[\mathrm{IC}_{95 \%}\right]}\end{array}$ \\
\hline \multirow[t]{2}{*}{ Sexo } & Hombres & $\begin{array}{c}216(78.3) \\
{[73.2-80.9]^{a *}}\end{array}$ & $\begin{array}{c}54(83.1) \\
{[73.5-87.2]^{\mathrm{as}}}\end{array}$ \\
\hline & Mujeres & $\begin{array}{c}60(21.7) \\
{[17.1-26.8]^{\text {bo }}}\end{array}$ & $\begin{array}{c}11(16.9) \\
{[8.9-27.8]^{6}}\end{array}$ \\
\hline \multirow[t]{4}{*}{$\begin{array}{l}\text { Agente causal } \\
\text { de la quemadura }\end{array}$} & Electricidad & $\begin{array}{c}71(25.7) \\
{[20.8-30.9]^{\mathrm{a}}}\end{array}$ & $\begin{array}{c}27(41.5) \\
{[30.0-52.4]^{b *}}\end{array}$ \\
\hline & Fuego directo & $\begin{array}{c}128(46.4) \\
{[40.5-51.4]^{\mathrm{a}}}\end{array}$ & $\begin{array}{c}29(44.6) \\
{[32.9-55.2]^{a *}}\end{array}$ \\
\hline & Agua caliente & $\begin{array}{c}39(14.1) \\
{[10.3-18.7]^{a}}\end{array}$ & $\begin{array}{c}4(6.2) \\
{[1.7-14.9]^{b *}}\end{array}$ \\
\hline & Otros & $\begin{array}{c}38(13.8) \\
{[10.0-18.3]^{\mathrm{a}}}\end{array}$ & $\begin{array}{c}5(7.7) \\
{[2.6-16.9]^{\text {as }}}\end{array}$ \\
\hline \multirow[t]{3}{*}{ Tipo de accidente } & Ocupacional & $\begin{array}{c}89(32.2) \\
{[26.9-37.6]^{\mathrm{at}}}\end{array}$ & $\begin{array}{c}23(35.4) \\
{[24.3-46.7]^{a s}}\end{array}$ \\
\hline & Domiciliar & $\begin{array}{c}65(23.6) \\
{[18.7-28.7]^{\mathrm{at}}}\end{array}$ & $\begin{array}{c}13(20.0) \\
{[11.3-31.2]^{\text {at }}}\end{array}$ \\
\hline & Otro & $\begin{array}{c}122(44.2) \\
{[38.4-49.3]^{\text {a }}}\end{array}$ & $\begin{array}{c}29(44.6) \\
{[32.9-55.2]^{\text {as }}}\end{array}$ \\
\hline \multirow[t]{2}{*}{ NSCQ } & Un segmento & $\begin{array}{c}88(32.2) \\
{[26.8-37.6]^{\mathrm{as}}}\end{array}$ & $\begin{array}{c}9(14.1) \\
{[6.7-24.7]^{b}}\end{array}$ \\
\hline & $>1$ segmento & $\begin{array}{c}185(67.8) \\
{[62.1-71.4]^{\mathrm{at}}}\end{array}$ & $\begin{array}{c}55(85.9) \\
{[79.9-89.5]^{\text {b* }}}\end{array}$ \\
\hline \multirow[t]{3}{*}{$P Q$} & Grado 1 & $\begin{array}{c}6(2.2) \\
{[0.8-4.7]^{*}}\end{array}$ & ** \\
\hline & Grado 2 & $\begin{array}{c}208(75.3) \\
{[70.1-78.3]^{a \mathrm{at}}}\end{array}$ & $\begin{array}{c}24(38.7) \\
{[27.1-50.1]^{\mathrm{b} *}}\end{array}$ \\
\hline & Grado 3 & $\begin{array}{c}62(22.5) \\
{[17.7-27.6]^{a \bullet}}\end{array}$ & $\begin{array}{c}38(61.3) \\
{[49.1-69.9]^{b *}}\end{array}$ \\
\hline $\begin{array}{l}\text { Los porcentajes correspo } \\
\text { representan los resultado } \\
\text { pruebas entre grupos y los } \\
\star \star \\
\text { No se documentaron II }\end{array}$ & $\begin{array}{l}\text { cuencias de cada v } \\
\text { as de hipótesis, con } \\
\text { as intragrupo. } \\
\text { aciente con quemad }\end{array}$ & $\begin{array}{l}\text { las columnas. La } \\
\text { omo estadistico d } \\
\end{array}$ & $\begin{array}{l}\text { signos en superín } \\
\text {-as letras represe }\end{array}$ \\
\hline
\end{tabular}

No observamos una tendencia en el tiempo respecto al número de ingresos por sexo (Fig. 4). Los pacientes sin IISQ presentaron una proporción mayor en hombres que en mujeres $(78.3 \%$ frente a $21.7 \%)$; de igual manera, observamos mayor proporción de hombres con IISQ que de mujeres $(83.1 \%$ frente a $16.9 \%)$. No observamos diferencias significativas entre infectados y no infectados en las frecuencias para el mismo sexo (Tabla III).

El agente causal más frecuente de las quemaduras, en pacientes sin o con IISQ fue el fuego directo, seguido por la electricidad, el agua caliente y otros (Fig. 5). Observamos diferencias significativas entre el agua caliente y la electricidad en los pacientes infectados y no 


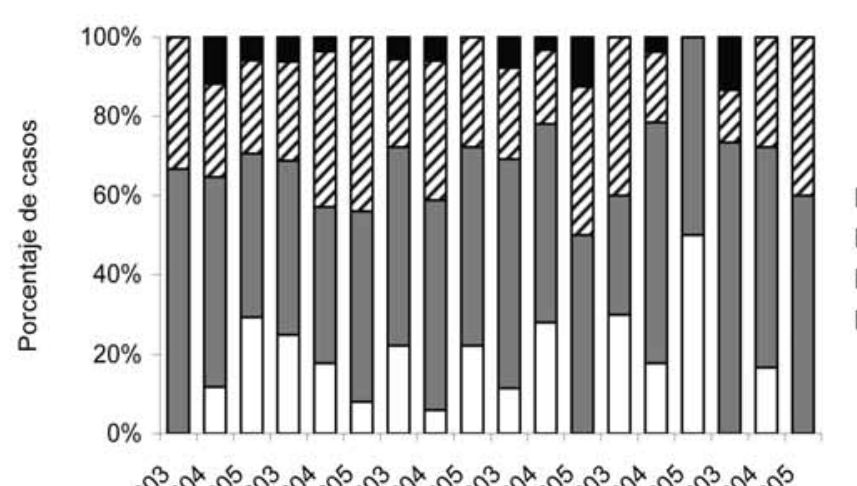

\section{- $>65$ años प40-64 años प20-39 años $\square 13$ a 19 años}

$\square$ Mujeres 口Varones
Fig. 3. Distribución de los ingresados por quemadura, según la edad, en la UNQ-HSJ D. Enero de 2003 a diciembre de 2005.

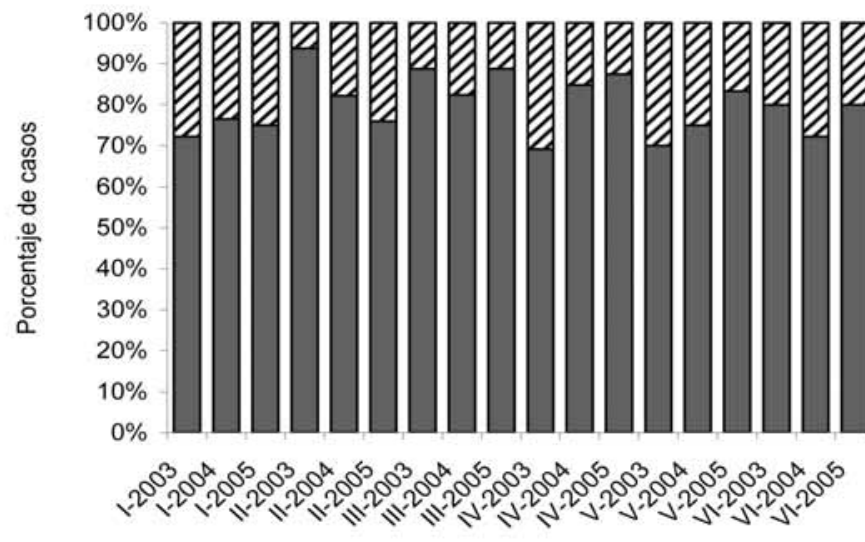

infectados, no así entre el fuego directo y otros agentes. En los pacientes sin IISQ no hubo diferencia proporcional entre el agua caliente y otros, como sí existió entre el agua caliente, la electricidad y el fuego directo. Para los pacientes con IISQ el fuego directo y la electricidad no presentaron diferencias, al igual que entre el agua caliente y otros agentes (Tabla III).

Las quemaduras por accidente ocupacional fueron más frecuentes que las quemaduras por accidentes domiciliares en pacientes con y sin IISQ. Cabe destacar que la categoría "otros" asumió un valor proporcional mayor que los accidentes ocupacionales (Fig. 6). Las proporciones entre los grupos de infectados y no infectados no presentaron diferencias significativas, mientras que sí se observaron intragrupos (Tabla III).

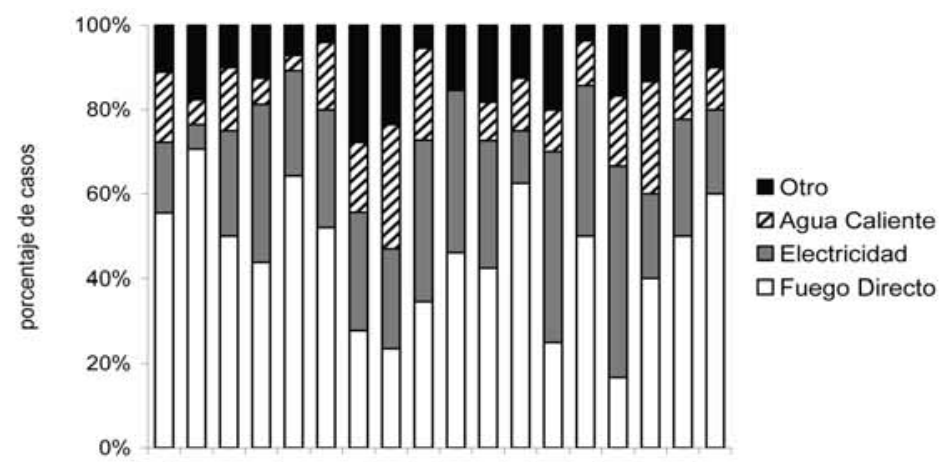

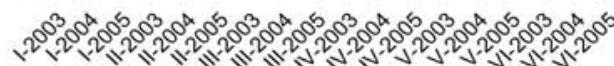

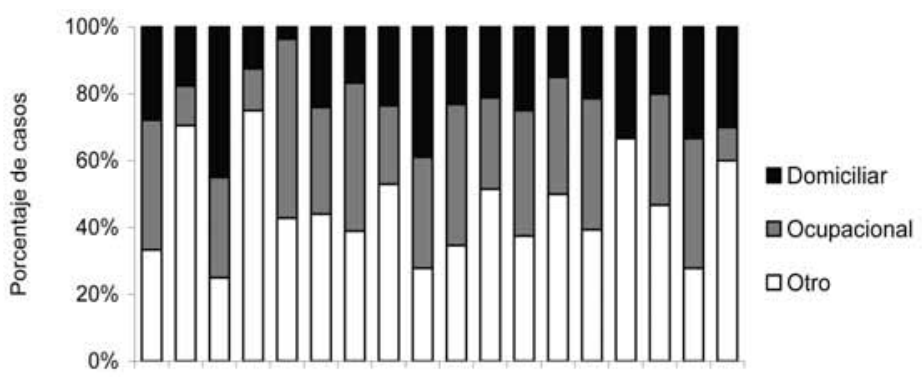

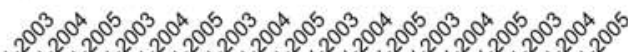

Fig. 5. Distribución de los ingresados por quemadura(s), según agente causal de la quemadura en la UNQ-HSJ D. Enero 2003 a diciembre 2005.
Fig. 6. Distribución de los ingresados por quemadura(s), según tipo de accidente que produjo la quemadura en la UNQ-HSJ D, Enero 2003 a diciembre 2005. 


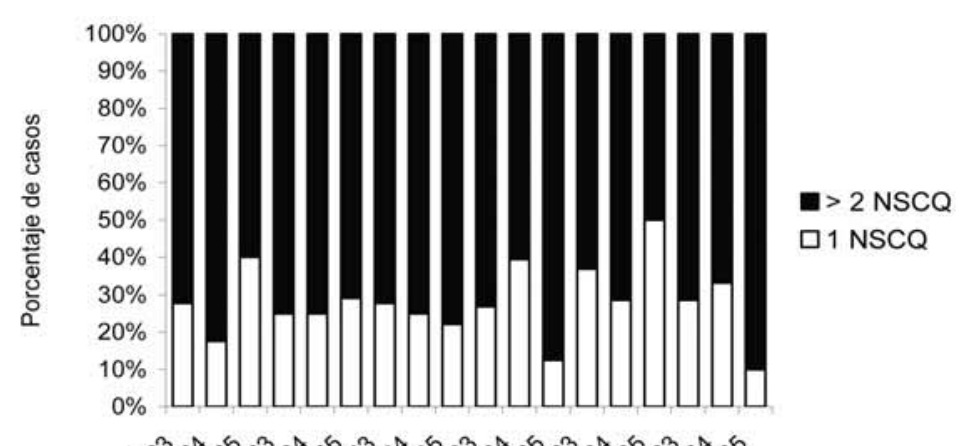

Fig. 7. Distribución de los ingresados por quemadura(s), según NSCQ en la UNQ-HSJ D según bimestre y año. Enero 2003 a diciembre 2005.

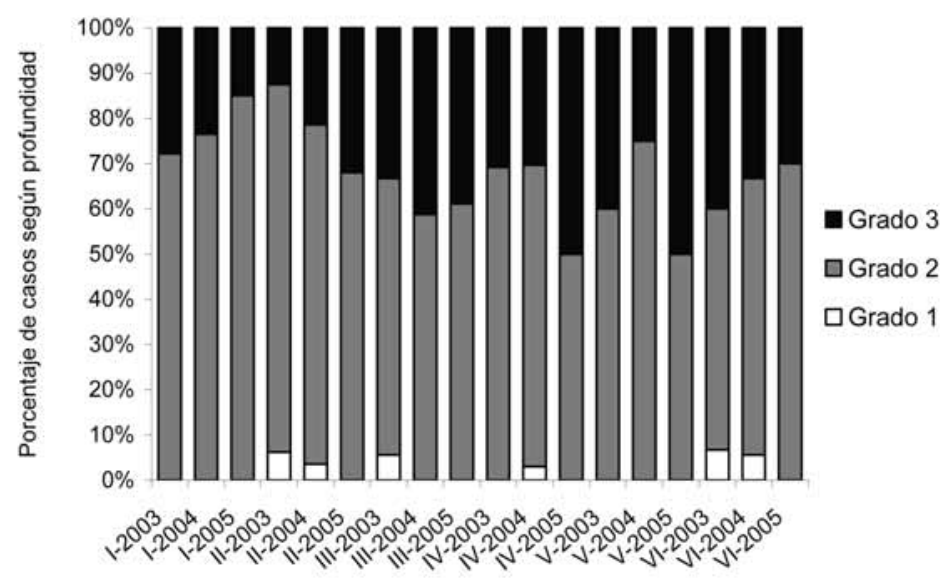

Fig. 8. Distribución de los ingresados por quemadura(s), según la PQ en la UNQ-HSJ D. Enero 2003 a diciembre 2005.

Según el NSCQ, observamos mayor proporción de IISQ en pacientes con más de un segmento corporal quemado. También que en el año 2005, en los bimestres I, II y V, aumentó la proporción de pacientes con un segmento corporal quemado (Fig. 7). Tanto para los pacientes con como sin IISQ, observamos que la mayoría presentaban dos o más segmentos corporales quemados (Tabla III).

Las quemaduras grados 2 y 3 predominaron en todos los años de estudio (Fig. 8), mientras que el grado 1 fue el menos frecuente y no se presentó en todos los bimestres. En los pacientes sin IISQ, observamos una mayor proporción de sujetos con quemaduras grado $2(75.3 \%)$ y en los pacientes con IISQ se observó una mayor proporción de sujetos con quemaduras grado 3 (63.1\%) (Tabla III).

\section{Discusión}

Los meses en que encontramos el mayor número de pacientes quemados fueron los posteriores al ingreso a clases escolares, ya fuera de principio o de medio año. Observamos que las estadísticas de quemaduras varían entre los diferentes países y que son imprecisas $(4,5)$, así como que las quemaduras dependen de las características socioculturales de cada pueblo $(2,3,7)$. Probablemente esa tendencia temporal en la presentación de las quemaduras se deba a actividades asocia- das a características sociales y culturales propias del país, como partidos de fútbol, períodos utilizados para viajes o momentos de recreo; o bien, períodos en los que los individuos están mayormente en sus casas de habitación o en sus trabajos. Ramakrishnan et al. (16) determinaron que el $68 \%$ de las quemaduras eléctricas se debían a descuidos de los trabajadores que no estaban atentos a sus quehaceres, y ocurrían principalmente tras el regreso vacacional a los centros de trabajo. A pesar de no encontrar una tendencia temporal en la aparición de las IISQ, sí vimos mayores tasas bimestrales de IISQ por cada 100 pacientes ingresados a la UNQ-HSJD en el año 2005. Durante ese mismo año, los profesionales médicos restringieron el ingreso a la UNQ-HSJD: solo fueron admitidos casos severos de quemaduras. Puede que esto haya influido en la disminución de ingresos observada en el año 2005, así como en el aumento de las tasas bimestrales de IISQ durante los tres últimos bimestres de ese año (Prada, 2006, comunicación personal).

Los pacientes que presentaron IISQ tuvieron un promedio de DEH mayor que los que no se infectaron. En general, los pacientes con IIH prolongan su estancia de 4 a 10 días más de lo esperado para su patología (9). Sin embargo, los pacientes quemados que presentaron IISQ tuvieron una estancia hospitalaria 3.8 veces mayor respecto a los pacientes que no desarrollan IISQ. Los DEHP-IISQ fueron en prome- 
dio 10.1 días; esta situación podría deberse a dos razones: una, al incremento en DEH que aumenta la exposición a las infecciones nosocomiales y la otra a que son pacientes más complicados desde su ingreso por ser sus quemaduras, por lo general, más profundas y abarcar mayor SCTQ.

La incidencia acumulada de IISQ encontrada en este estudio es menor con respecto a la referida por los profesionales que atienden la UNQ-HSJD (19/100 ingresos frente a 40/100 ingresos). Es probable que la alta incidencia recogida por los responsables de la UNQ-HSJD esté sobreestimada, pues toma en cuenta criterios como las alteraciones en el hemoleucograma (Fonseca, 2006, comunicación personal), que no fueron considerados en este estudio. Una característica de la definición de caso del estudio, fue que el médico debía hacer el diagnóstico de IISQ y consignarlo en el expediente clínico. Impresionó el hecho de que se iniciaran tratamientos antimicrobianos en pacientes que presentaban alteraciones en su hemoleucograma, pero en los que no se estableció el diagnóstico de IISQ en el expediente clínico; a otros pacientes, pese a presentar alteraciones en el hemoleucograma, no se les inició tratamiento antimicrobiano. Por tanto, no están estandarizadas las observaciones del personal que brinda atención a los pacientes de la UNQ-HSJD en relación al hemoleucograma y la IISQ.

En el presente estudio, era factible encontrar que las quemaduras grado 2 y 3 fueran las más frecuentes debido a que la UNQ-HSJD admite únicamente grandes quemados y no las quemaduras más leves (grado 1). Al igual que en la literatura (5-7), la mayor proporción de infectados apareció en pacientes con quemaduras grado 3. Adicionalmente, los pacientes infectados presentaron un promedio mayor de SCTQ. Observamos que las tasas de infección en el paciente quemado se incrementaron de manera proporcional respecto a la SCTQ y la PQ, de manera que todos los pacientes con quemaduras graves sufrirán, con una altísima probabilidad, una infección. La cantidad de tejido muerto es el indicador determinante para el desarrollo de procesos infecciosos $(9,17)$.

La electricidad produjo la mayor proporción de pacientes quemados con IISQ; ésta, al pasar por el cuerpo, se convierte en calor fuera del mismo y produce una quemadura de llamarada. Cuando esta conversión ocurre dentro del cuerpo, el calor se incrementa por la resistencia ofrecida por algunas estructuras como el hueso, dañando así los tejidos y causando una necrosis térmica. El daño térmico sostenido por esta flama, afecta la vestidura y la enciende (16), lo que explica el gran proceso necrótico sufrido por los pacientes con quemaduras eléctricas, y por tanto, su susceptibilidad para desarrollar infecciones. Es nece- sario indicar que las lesiones térmicas de la piel se siguen del desarrollo inmediato de edema con alto contenido de proteínas y líquidos, asociado con necrosis de grado variable según la profundidad de la lesión (9). Los pacientes que sufrieron quemaduras por agua caliente desarrollaron menos infecciones, posiblemente, porque el agua no afectó a grandes superficies corporales y el daño fue menos profundo.

Hubo diferencias en el desarrollo de IISQ entre sujetos de diferentes edades. Esto concuerda con lo presentado en otros estudios que documentan diferencias en la susceptibilidad a la IISQ según la edad del paciente $(8,9)$. En nuestro estudio determinamos una mayor proporción de IISQ en los pacientes mayores de 64 años de edad (28.6\%). No observamos que el sexo y el tipo de accidente influyeran en la presentación de IISQ; no obstante, se presentó una mayor proporción de hombres quemados, lo que concuerda con otros trabajos $(2,6)$. Si bien no hay una explicación clara del porqué de esta situación, podría deberse a los roles que los géneros cumplen en las comunidades. Se explica así la tendencia a creer que los accidentes ocupacionales son más frecuentes que los domiciliares; en otras palabras, en nuestra sociedad los hombres son, por lo general, los principales proveedores en la economía familiar y están involucrados, principalmente, en ocupaciones en las que un descuido podría generar un accidente laboral cuyo desenlace podría ser una quemadura, por ejemplo, los electricistas y los trabajadores de gasolineras.

\section{Conclusiones}

La destrucción de tejidos y el ambiente rico para el crecimiento bacteriano producto de las quemaduras, junto con las alteraciones en el sistema inmunológico, hacen que los pacientes quemados tengan una incidencia acumulada de infección intrahospitalaria mayor que la reportada en los centros hospitalarios, para los pacientes en general.

Las características propias de la quemadura por electricidad, así como la profundidad de la quemadura y la superficie corporal quemada alcanzadas por este agente, favorecen una mayor probabilidad de presentar infección intrahospitalaria en el sitio de la quemadura que cuando se trata de otros agentes causales.

Nuestra artículo provee elementos que permiten identificar factores relativos al individuo y a las características de la quemadura que deben ser tomados en cuenta por los responsables de las Unidades de Quemados para identificar aquellos pacientes con posibilidades incrementadas de sufrir infección intrahospitalaria en el sitio de la quemadura y así poder controlar y prevenir este tipo de infección y sus efectos. 


\section{Agradecimientos}

A los médicos asistentes del Hospital San Juan de Dios, que ayudaron a la revisión de expedientes clínicos: Ronald Gutiérrez Cerdas y Herman Morera Hidalgo.

\section{Dirección del autor}

\section{Dr. Sócrates Vargas Naranjo}

Urb. Jardines del Beneficio. Apto 2 Beige entre calle 2 y av. 4. Santa Lucía de Barva de Heredia. Costa Rica.

e-mail:socrates_vargas@yahoo.it

\section{Bibliografía}

1. En: www.sos-urgencias.iespana.es/sos-urgencias/quemaduras.htm

2. En:www.medilegis.com/BancoConocimiento/T/TM103N9_ ABP1/TM103N9_ABP1.osp

3. De-Souza, D.A., Marchesan, W.G., Greene, L.J.: "Epidemiological data and mortality rate of patients hospitalized with burns in Brazil". Burns 1998; 24:433.

4. Pereira, C.T., Barrow, R.E., Sterns, A.M., Hawkins, H.K., Kimbroungh, C.W., Jeschke, M.G., Lee, J.O., Sanford, A.P. and Herndon, D.N.: "Age-dependent differences in Survival after Severe Burns: A Unicentric Review of 1,674 Patients and 179 Autopsies over 15 years". American Collage of Surgeons 2006; 202:536.
5. Ponce de León, R. S. y Soto H, J. L.: "Infecciones intra hospitalarias”. McGraw-Hill-Interamericana, México D. F. 1996.

6. En: www.indexer.net/quemados

7. Goldman, S., Limor, A. D.: "The Israel Trauma Group and Peleg, K.. Childhood burns in Israel: A 7 year epidemiological review". Burns 2006; 32:467.

8. Dávila Alfaro, M.: "Quemaduras". EDNASSS. San José Costa Rica. 2005.

9. Rodríguez Menés, H., Rico Aguado, A. y del Pinto Paredes, V.: "Epidemiología, prevención y tratamiento de las quemaduras eléctricas infantiles". Cir.plást. iberolatinoam. 1988;14:265-8

10. Taneja, N., Emmanuel, R., Chari, P.S. and Sharma, M.: “A prospective study of hospital-acquired infections in burn patients at a tertiary care referral center in North India". Burns 2004;30:665-9.

11. Ponce de León R. S.: "Manual de Prevención y Control de Infecciones Intra Hospitalarias”. OPS-OMS. 1996.

12. Malagón-Londoño, G. y Hernández Esquivel, L.: "Infecciones hospitalarias". Editorial Médica Panamericana. Bogotá Colombia. 1995.

13. Nakhla, L.S. and Sanders, R.: "Microbiological aspects of burns at Mount Vernon Hospital, UK". Burns 1991; 17:309.

14. Mzezewa, S., Jönsson, K., Sibanda, E., Aberg, M. And Salemark, L.: "HIV infection reduces skin graft survival in burn injuries: a prospective study". The British Association of Plastic Surgeons 2003; 56:740.

15. Poindexter, B. J., Bhat, S., Buja, L.M., Bick, R.J. and Milner, S.M.: "Localization of antimicrobial peptides in normal and burned skin”. Burns 2006;32(4):402.

16. Ramakrishnan, K. M., Ramachandran, K., Jayaraman, V. and Mathivanen, T.: "Electrical burns treated in an Indian Hospital". Burns 1991; 17:481.

17. Stratta, R. J., Warden, G. D., Ninnemann, J. L. and Saffle, J. R.: "Immunologic Parameters in Burned Patients: Effect of Therapeutic Interventions". The Journal of Trauma 1986; $26: 7$. 